\title{
Estimulação elétrica neural transcutânea (tens) na paroxismia do tronco cerebral: relato de caso
}

\author{
Transcutaneous electrical nerve stimulation \\ (tens) in brain stem paroxysms: case report
}

\author{
Eliane Maria Dias von Söhsten Lins ${ }^{1}$ \\ Catarina Costa Boffino ${ }^{2}$ (1) \\ Dábila Caroline Pandolfi Mantovani ${ }^{3}$ (1) \\ Flavia Paiva dos Santos Pereira 4 (1) \\ Edmo Arruda Aguiar Sobreira da Silveira ${ }^{5}$ (1)
}

${ }^{1}$ Autora para correspondência. Serviço de Otorrinolaringologia, Hospital Santa Marcelina (São Paulo). São Paulo, Brasil. elianesohsten@gmail.com Universidade Metodista de São Paulo (São Paulo). São Paulo, Brasil. boffinocb@gmail.com 3-5Serviço de Otorrinolaringologia, Hospital Santa Marcelina (São Paulo). São Paulo, Brasil. dabilacpm@hotmail.com, flapaiva@gmail.com, edmoarruda@gmail.com

RESUMO | INTRODUÇão: A paroxismia de tronco encefálico, descrita em pacientes com esclerose múltipla (EM), caracteriza-se por episódios rápidos de vertigem e nistagmos evocados por movimentos cefálicos. A neuromodulação tem apresentado sucesso terapêutico em várias disfunções da EM, mas ainda não foi utilizada na paroxismia. OBJETIVo: Avaliar o efeito aditivo da neuromodulação por estimulação elétrica neural transcutânea (TENS) na musculatura periocular em paciente com EM e paroxismia de tronco encefálico não responsiva à reabilitação clássica associada à medicação. MATERIAL E MÉTODOS: Avaliação do nistagmo com videonistagmógrafo, da sensibilidade cutânea facial com estesiômetro, do blefaroclônus por gravação em vídeo e da sensação dolorosa de forma subjetiva (descrição pessoal da paciente); execução de 10 sessões consecutivas semanais de exercícios oculares associados à TENS na musculatura do oblíquo inferior e reto lateral direitos. RESULTADO: Melhora da algia, do blefaroclônus, da vertigem e do nistagmo ocular direito. CONCLUSÃO: A neuromodulação com TENS parece ser uma terapia complementar válida para pacientes com EM e paroxismia de tronco não responsivos aos demais tratamentos clínicos, porém mais estudos são necessários para confirmar esse achado.

PALAVRAS-CHAVE: Esclerose múltipla. Nistagmo. Neuromodulação.
ABSTRACT | INTRODUCTION: Brainstem paroxysmia has been described in patients with multiple sclerosis, characterized by rapid episodes of nystagmus and vertigo evoked by cephalic movements. Neuromodulation has shown therapeutic success for several dysfunctions of multiple sclerosis, but it has not yet been used in paroxysmia. OBJECTIVE: To assess the additive effect of neuromodulation by transcutaneous electrical neural stimulation (TENS) on the periocular musculature in a patient with multiple sclerosis and brain stem paroxysmia that is not responsive to classical rehabilitation associated with medication. MATERIAL AND METHODS: Nystagmus evaluation with video nystagmography, facial skin sensitivity analysis with esthesiometer, video recording of blepharoclonus, and pain sensation subjectively quantification (by a personal description of the patient); execution of 10 eyes consecutive exercises sessions associated with TENS in the right lower oblique and lateral rectus muscles. RESULT: Improvement in pain, blepharoclonus, and right eye nystagmus. CONCLUSION: Neuromodulation with TENS seems to be a valid complementary therapy for patients with brainstem paroxysmia unresponsive to other clinical treatments, but more studies are needed to confirm this finding.

KEYWORDS: Multiple sclerosis. Nystagmus. Neuromodulation. 


\section{Introdução}

Esclerose múltipla (EM) é uma doença inflamatória crônica e desmielinizante de origem autoimune. É caracterizada por lesões multifocais e temporárias no sistema nervoso central (SNC) com dano axonal. Acomete principalmente adultos jovens, do sexo feminino, e ocasiona grande impacto psico-social. ${ }^{1}$ A desmielinização ocorre frequentemente no cérebro e cerebelo; portanto, sensações vestibulares anormais como vertigem e desequilíbrio são comuns em seu curso clínico. Cerca de 78\% dos pacientes apresentam anormalidades do equilíbrio, 18-63\% apresentam nistagmo, e a vertigem verdadeira é encontrada em mais de $17 \% . \frac{3}{\text { Raros sintomas incluem }}$ convulsões, perda auditiva e paralisias. ${ }^{4}$ A paroxismia de tronco foi inicialmente descrita, em 1925, por Carmichael e Critchley como "nistagmos faciais" deflagrados no olhar lateral em pacientes com EM. ${ }^{5}$

O manejo dos sintomas da EM inclui métodos não farmacológicos como reabilitação e suporte psicológico, medicações e procedimentos cirúrgicos. ${ }^{4}$

A reabilitação vestibular é uma terapia de suporte para pacientes com distúrbios do equilíbrio. Tem excelente resposta nas vestibulopatias periféricas. Já nos pacientes com alterações centrais, como é o caso dos com EM, os resultados parecem menos expressivos. ${ }^{6}$

O TENS tem sido amplamente usado para alívio da dor, envia aferências somatossensorias adicionais, diminuindo a percepção dolorosa. Seu uso na dor foi avaliado tanto em modelos animais quanto em experiências com humanos. Em alta frequência e baixa intensidade gera uma sensação de formigamento não dolorosa, e em baixa frequência e alta intensidade, ativa fibras aferentes nociceptivas de pequeno diâmetro, A\& e C, resultando em uma sensação dolorosa, mas tolerável. Essas aferências são capazes de estimular estruturas a nível do sistema nervoso central, neuromodulando-as e gerando analgesia. $\underline{. \underline{\underline{8}}}$

A neuromodulação tem se expandido em várias subespecialidades neurológicas. Desordens do movimento e síndromes dolorosas após acidentes vasculares encefálicos estão entre as indicações neurológicas mais comuns. Em EM, a bomba de baclofeno intratecal tem sido a principal técnica de neuromodulação utilizada por muitos pacientes para melhorar o manejo da espasticidade. ${ }^{9}$ Não há descrição do uso da neuromodulação para os sintomas da paroxismia de tronco em pacientes com EM, razão do nosso interesse em relatar o caso.

\section{Objetivo}

Relato de um caso de reabilitação vestibular associada à neuromodulação com TENS em paciente com EM e paroxismia de tronco.

\section{Material e Métodos}

Protocolo de número 32886620.4.0000.0066 aprovado na Plataforma Brasil.

Paciente assinou termo de consentimento e termo de autorização para uso de imagens.

Realizou-se videonistagmografia (VisualEyes525, InteracousticsR) (VNG) para análise e quantificação dos nistagmos e estesiometria facial antes do início da neuromodulação, ao término e um mês após.

Utilizou-se o TENS (TENS 3000, portátil, EUA) configurado com uma frequência de $70 \mathrm{~Hz}$, duração de pulso de $120 \mu \mathrm{s}$, modo N-Pulse, que significa estimulação constante. O modo mostra a programação ao qual o aparelho apresenta a corrente. A taxa de pulso é automaticamente variada em um padrão cíclico ao longo de um intervalo de normalmente 10 segundos (no máximo $150 \mathrm{~Hz}$ ). A taxa de pulso decresce linearmente ao longo de um período de 4 segundos, a partir do controle do valor de ajuste, para um valor que é $40 \%$ menos. A taxa de pulso mais baixa vai continuar durante 1 segundo. Depois vai aumentar linearmente durante um período de 4 segundos até o seu valor original. A taxa de pulso original irá continuar por 1 segundo. O ciclo é depois repetido. A forma da onda é assimétrica, bifásica com pulso quadrado. Estimulação contínua na musculatura do reto lateral e do oblíquo inferior do olho direito por 40-50 minutos. Os eletrodos foram colocados na face, musculatura extraocular, próximo ao ventre da musculatura alvo (figura 1). 
Figura 1. Posicionamento dos eletrodos

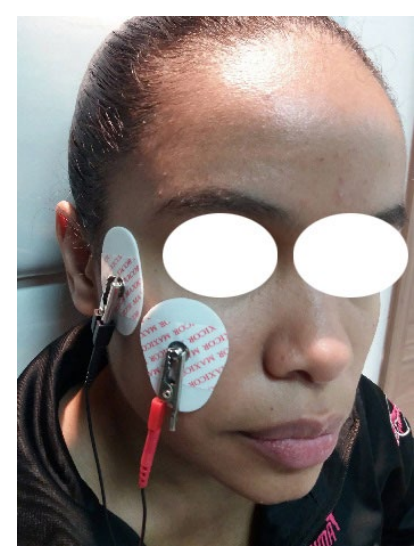

A intensidade do aparelho foi regulada de acordo com a percepção da paciente e, geralmente, correspondia a uma sensação de vibração na musculatura extraocular e leve contração desta. Iniciava-se a primeira parte da terapia apenas com o estímulo do TENS sem associação do exercício. Nos últimos 10 a 15 minutos, associavase os exercícios de reflexo vestíbulo-ocular clássicos, de acordo com os protocolos de reabilitação vestibular (Cawthorne e Cooksey, e Herdman). Foram prescritos os mesmos exercícios para casa, 2 a 3 vezes ao dia, além de exercícios para treino do reflexo vestíbulo-ocular no computador, ambos sem associação com TENS. Iniciou-se com exercícios horizontais, progredindo para verticais.

\section{Relato de Caso}

SFO, sexo feminino, 25 anos, há 7 anos (05/2012) teve diagnóstico de EM forma surto-remissão. Apresentou episódios recorrentes de paralisia de hemidimídio direito, hemiface direita, hemidimídio esquerdo, afasia, diplopia, vertigens, náuseas e vômitos; perda de peso (11 kg em 5 meses) e surdez súbita na orelha direita. Ressonância do crânio com detecção de lesões desmielinizantes acometendo região periventricular, cerebelo, pedúnculos médios, ponte, bulbo, corpo caloso, segmentos medulares de C2 a C6 (figuras 2 e 3) e análise de líquor com resultado compatível com EM. Audiometria de tronco encefálico em 02/13 com limiares de $60 \mathrm{~dB}$ na orelha esquerda e 105 dB na orelha direita, com aumento das latências absolutas das ondas III e V e das latências interpicos I-V e III-V bilateralmente. Em 01/17, após surto bilateral, perdeu força dos 4 membros e diminuiu a audição do lado direito, passou a ter vertigem súbita rápida e vertigem e zumbido induzidos pela rotação da cabeça para direita ou pela lateralização do olhar para direita, dor e blefaroclônus no olho direito. Audiometria de 01/18 evidenciou normoacusia esquerda e perda auditiva neurossensorial moderada a severa na orelha direita. Audiometria de tronco encefálico de 04/18 detectou limiar de $105 \mathrm{~dB}$ na orelha direita e $25 \mathrm{~dB}$ na orelha esquerda. Ressonância de condutos auditivos internos (03/18) normal. Teve diagnóstico de paroxismia de tronco encefálico e foi medicada com carbamazepina na dose de $100 \mathrm{mg}$, duas vezes ao dia, mas paciente só tolerou $100 \mathrm{mg}$ à noite, iniciou em 05/18. Com a medicação, houve considerável melhora dos sintomas da paroxismia, mas persistência do blefaroclônus e da dor ocular direita. Ao exame, apresentava nistagmo espontâneo torcional, anti-horário, para direita, com olhos abertos, que aumentava de intensidade na lateralização do olhar para direita. Tinha diminuição da sensibilidade tátil da hemiface direita e leve diminuição da força de membro inferior esquerdo, sem prejuízo funcional. 
VNG (07/18) com alterações sugestivas de lesão vestibular de etiologia central (nistagmo espontâneo torcional anti-horário, com componente horizontal para direita e vertical para baixo de 5\%s cada; calibração, perseguição, sacadas e optocinético alterados; intensificação do nistagmo espontâneo em Rose bilateral e na torsão bilateral; preponderância direcional do nistagmo para direita na prova calórica com ar). Em 07/18, iniciou reabilitação vestibular com exercícios para otimização dos reflexos vestíbulo-oculares horizontal e vertical e foi proposta terapia experimental com neuromodulação TENS direcionada ao alívio do blefaroclonus e da dor periocular, bem como, reforço no controle do nistagmo. Em 09/18, teve dor intensa na região malar direita, irradiada para orelha direita. Quando a dor cessou, notou retorno da audição nessa orelha. Realizou nova audiometria, que confirmou a normoacusia. Fez 10 sessões consecutivas de reabilitação vestibular clássica para otimizar o reflexo vestibuloocular horizontal associada ao TENS com intervalo semanal, apresentando melhora do nistagmo (a componente horizontal reduziu para $1 \% \mathrm{~s}$ ), a vertical continuou $5 \%$, normalização da sensibilidade tátil da face e cessação do blefaroclônus e da algia periocular. Redução de $80 \%$ do nistagmo horizontal. Foi orientado realizar exercícios domiciliares para melhora do reflexo vestíbulo-ocular vertical, mantendo os exercícios para o horizontal. Após um mês da última sessão, continuava bem, com a componente vertical do nistagmo menor ( $2 \%$ s) e a horizontal com a mesma velocidade $(1 \% \mathrm{~s})$.

Figura 2 e 3. Lesões desmielinizantes em região periventricular, cerebelo, pedúnculo médio, ponte e medula
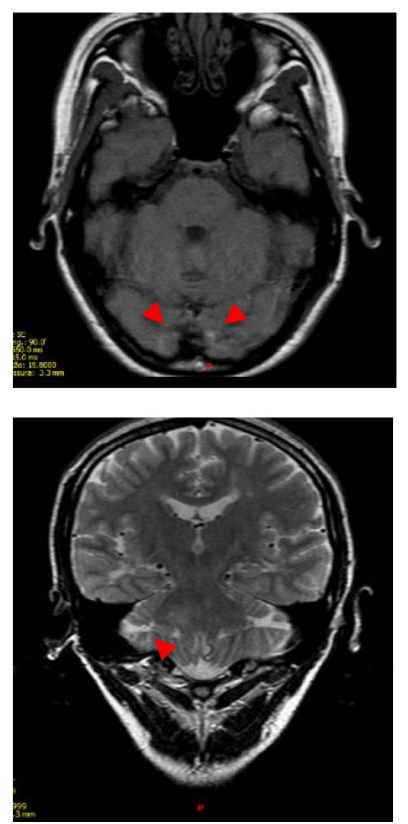

Quadro 1. Evolução clínica paciente

\begin{tabular}{|c|c|c|c|c|c|}
\hline Sinal/Sintoma & Blefaroclônus & $\begin{array}{l}\text { Nistagmo } \\
\text { Velocidade } \\
\text { Angular }\end{array}$ & Estesiometria face & $\begin{array}{l}\text { Algia } \\
\text { periocular } \\
\text { direita }\end{array}$ & Déficit auditivo \\
\hline Pré tratamento & Moderado & $\begin{array}{l}5 \% / \mathrm{s} \\
\text { horizontal } \\
5 \% / \mathrm{s} \\
\text { vertical }\end{array}$ & $\begin{array}{l}\text { Hemiface direita: } \\
\text { percepção tátil filamento } \\
\text { vermelho }(4 \mathrm{~g}) \\
\text { Hemiface esquerda: } \\
\text { percepção tátil filamento } \\
\text { azul } \\
(0,02 \mathrm{~g})\end{array}$ & Intensa & $\begin{array}{l}\text { Perda auditiva } \\
\text { moderada } \\
\text { a severa orelha } \\
\text { direita } \\
\text { Normo- } \\
\text { acusia } \\
\text { esquerda }\end{array}$ \\
\hline Pós tratamento & Cessou & $\begin{array}{l}1 \% \text { s } \\
\text { horizontal } \\
2 \% \mathrm{~s} \\
\text { vertical }\end{array}$ & $\begin{array}{l}\text { Oscilou durante } \\
\text { tratamento, teve } \\
\text { momentos com } \\
\text { hiperestesia a direita, mas } \\
\text { no final, manteve padrão } \\
\text { similar ao início }\end{array}$ & Cessou & $\begin{array}{l}\text { Normo- } \\
\text { acusia } \\
\text { bilateral }\end{array}$ \\
\hline
\end{tabular}




\section{Discussão}

Um prejuízo nos movimentos oculares, ou nistagmos, são vistos em muitas doenças do

SNC, em particular naquelas afetando o tronco-encefálico e cerebelo.

Keane, em 1978, descreveu dois casos de pacientes com doença desmielinizante que exibiam contraturas faciais dolorosas repetidas similares precipitadas pelo olhar excêntrico, envolvendo principalmente a musculatura orbicular dos olhos. Esses problemas palpebrais flutuavam em intensidade, mas persistiram por anos e não responderam à fenitoína ou a um curso breve de tratamento com carbamazepina. ${ }^{5}$

Quiles et al. em 1980, relataram três casos de EM que apresentaram durante seu curso, ataques paroxísticos, também denominados convulsões do tronco encefálico. $\frac{10}{}$

No tratamento medicamentoso do nistagmo, costuma-se utilizar os bloqueadores do canal de potássio para o down beat nistagmo, o baclofeno para o nistagmo alternante, gabapentina e memantina para nistagmo pendular adquirido, e a carbamazepina para a mioquimia do oblíquo superior. ${ }^{11}$ Tratamentos com essas drogas têm mostrado boa resposta em relação ao controle do nistagmo, com poucos efeitos colaterais, embora a recomendação do uso das mesmas tenha apenas nível de evidência C. 12

Além do tratamento farmacológico do nistagmo, outras abordagens terapêuticas foram e são utilizadas por diferentes áreas médicas. Na oftalmologia, procedimentos ortópticos, cirúrgicos são opções. Ciuffreda $\mathrm{KJ}$ et al. realizaram um treinamento de biofeedback auditivo para controlar nistagmo em cinco pacientes com nistagmo congênito e obtiveram uma redução média de $82 \%$, $86 \%$ e $34 \%$ da amplitude, pico da velocidade lenta e frequência do nistagmo, respectivamente. ${ }^{13}$

A paroxismia de tronco encefálico, evento secundário a lesão desmielinizante da EM ou a acidente vascular de tronco encefálico, entra no diagnóstico diferencial da paroxismia vestibular. A paroxismia vestibular é uma disfunção que se caracteriza por crises rápidas de vertigem, nistagmos e, às vezes, zumbido, deflagradas pela rotação cefálica, que se repetem várias vezes ao longo do dia; têm como mecanismo fisiopatológica descargas neurais efáticas originárias da compressão vascular na porção proximal do oitavo nervo craniano; apresenta excelente resposta ao tratamento com carbamazepina/oxcarbazepina. ${ }^{14}$

Na EM, a carbamazepina é utilizada para o tratamento de certos tipos de convulsões e de neuralgias, como a dor facial aguda, em choque, causada pela neuralgia do trigêmeo. $\frac{15}{}$

Outra modalidade de tratamento na EM é o uso da neuromodulação. 9 A atividade neuronal regula os oligodendrócitos e a mielinização axonal pode rapidamente modelar o diâmetro axonal, promover a reentrada de células progenitoras de oligodendrócitos dentro do ciclo celular, ou direcioná-las para diferenciação em oligodendrócitos. $\frac{16}{}$

Vários autores descrevem casos de surdez súbita em pacientes com EM com boa evolução em relação a recuperação auditiva. ${ }^{17}$ Nossa paciente apresentou uma forma incomum de manifestações da EM, com surdez súbita e evolução para episódios de paroxismia de tronco encefálico, foi submetida a um tratamento não usual, razão pelo nosso interesse em relatar o seu caso. Foi submetida a um tratamento experimental com neuromodulação associada à reabilitação vestibular.

Como já é descrito a efetividade da carbamazepina na melhora de alguns sintomas neurológicos de pacientes com EM ${ }^{15}$, bem como ser uma droga com excelente resposta na paroxismia vestibular, disfunção também decorrente de uma descarga efática ${ }^{14}$, optou-se pelo tratamento medicamentoso do nistagmo com esse fármaco, porém a paciente só tolerou uma dose baixa e não apresentou melhora completa do nistagmo, blefaroclônus e da algia periocular direita.

Por se tratar de uma doença com acometimento do SNC, onde a resposta à reabilitação clássica não é tão eficaz como nas disfunções periféricas ${ }^{\underline{6}}$ e pela presença da dor periocular e do blefaroclônus, optou-se pela associação da neuromodulação ao tratamento.

Sendo assim, a terapia experimental com o TENS foi idealizada para cumprir dois propósitos: o primeiro, o alívio da dor e o segundo, diminuir o nistagmo e tentar auxiliar na compensação. 
A teoria da otimização da recuperação neuronal com aceleração da mielinização induzida pela estimulação das vias neuronais fornece uma base fisiológica para a proposta de neuromodulação em pacientes com EM. $\frac{16}{}$

A paciente apresentou uma excelente resposta clínica ao tratamento, mas não podemos afirmar qual ou quais dos tratamentos (medicação, reabilitação vestibular clássica, neuromodulação) utilizados foi ou foram responsáveis pelo sucesso terapêutico, nem se decorreu da melhora por remielinização espontânea.

A sua boa recuperação audiológica está de acordo com outros casos já descritos. ${ }^{17}$

O fato da rápida melhora, após 10 semanas, com resolução da algia periocular, do blefaroclônus e diminuição do nistagmo, sintomas presentes já há um ano e meio, leva-nos a acreditar que a neuromodulação possa ter tido algum papel nesse processo.

\section{Conclusão}

Relato de caso não usual de EM, com grande riqueza de sintomas (paroxismia de tronco, surdez súbita, hipoestesia de hemiface direita) e sua boa resposta ao tratamento com carbamazepina associada à reabilitação vestibular clássica e à neuromodulação periférica com TENS. Houve desaparecimento da dor periocular e do blefaroclônus direitos; normalização da audição direita; e redução da velocidade angular (componente horizontal em $80 \%$ e vertical em $60 \%$ ) do nistagmo torcional presente no olho direito. A evolução do caso é sugestiva de que a neuromodulação teve um efeito pelo menos aditivo às outras modalidades terapêuticas. Trabalhos similares são necessários para validar esses achados.

\section{Contribuições dos autores}

Lins EMDS fez o diagnóstico e tratamento clínico, concebeu a neuromodulação alternativa para a paciente, participou da revisão bibliográfica e da confecção do texto. Boffino CC concebeu a neuromodulação alternativa para a paciente, executou o programa de reabilitação vestibular clássica e associada à neuromodulação, participou da revisão bibliográfica e da confecção do texto. Mantovani DCP e Pereira FPS auxiliaram na captação dos exames complementares e na revisão bibliográfica. Silveira EAAS auxiliou no diagnóstico clínico e na revisão bibliográfica.

\section{Conflitos de interesses}

Nenhum conflito financeiro, legal ou político envolvendo terceiros (governo, empresas e fundações privadas, etc.) foi declarado para nenhum aspecto do trabalho submetido (incluindo, mas não se limitando a subvenções e financiamentos, participação em conselho consultivo, desenho de estudo, preparação de manuscrito, análise estatística, etc.).

\section{Referências}

1. Mc Donald I. Diagnostic methods and investigations in multiple sclerosis. In: Compston A, Ebers G, Lassmann H, Mc Donald I, Matthews B, Wekerle $H$, editores. McAlpine's multiple sclerosis. 3a. ed. Nova lorque: Churchill Livengstone; 1999.

2. Frohman EM, Kramer PD, Dewey RB, Kramer L, Frohman TC. Benign paroxysmal positioning vertigo in multiple sclerosis: diagnosis, pathophysiology and therapeutic techniques. Mult Scler. 2003;9(3):250-5. https://doi. org/10.1191/1352458503ms9010a

3. Willian NP, Roland PS, Yelli W. Vestibular evaluation in patients with early multiple sclerosis. Am J Otol 1997;18(1):93-100. Citado em: PMID: 8989958

4. Ben-Zacharia AB. Therapeutics for multiple sclerosis symptoms. Mt Sinai J Med. 2011;78(2):176-91. https://doi.org/10.1002/ $\underline{\mathrm{msj} .20245}$

5. Keane JR. Gaze-evoked blepharoclonus. Ann Neurol. 1978;3(3):243-5. https://doi.org/10.1002/ana.410030310 
6. Bittar RSM, Pedalini MEB, Ramalho JO, Yoshimura R. Análise crítica dos resultados da reabilitação vestibular em relação à etiologia da tontura. Rev Bras Otorrinolaringol. 2007;73(6):760-4. http://dx.doi.org/10.1590/50034-72992007000600007

7. Bouhassira D, Bars D, Villanueva L. Heterotopic activation of A delta and $C$ fibres triggers inhibition of trigeminal and spinal convergent neurones in the rat. J. Physiol. 1987;389:301-17. https://doi.org/10.1113/jphysiol.1987.sp016658

8. Peng WW, Tang ZY, Zhang FR, Li H, Kong YZ, lannetti GD, et al. Neurobiological mehanisms of TENS-induced analgesia. Neurolmage. 2019;195:396-408. https://doi.org/10.1016/j. neuroimage.2019.03.077

9. Abboud H, Hill E, Siddiqui J, Serra A, Walter B. Neuromodulation in multiple sclerosis. Mult Scler. 2017;23(13):1663-76. https://doi. org/10.1177/1352458517736150

10. Llisterri AQ, Borrego FR, Serra JP. Paroxymal phenomena in multiple sclerosis. Brain stem crisis. Acta Neurol Latinoam. 1980;26(1):1-11. Citado em: PMID: $\underline{7246047}$

11. Straube A. Pharmacology of vertigo/nystagmus/ oscillopsia. Curr Opin Neurol. 2005;18(1):11-4. https://doi. org/10.1097/00019052-200502000-00004
12. Straube A. Nystagmus: an update on treatment in adults. Expert Opin Pharmacother. 2005;6(4):583-90. https://doi. org/10.1517/14656566.6.4.583

13. Ciuffreda KJ, Goldrich SG, Neary C. Use of eye movement auditory biofeedback in the control of nystagmus. Am J Optom Physiol Opt. 1982;59(5):396-409. https://doi. org/10.1097/00006324-198205000-00007

14. Strupp M, Lopez-Escamez JA, Kim JS, Straumann D, Jen JC, Carey J, et al. Vestibular paroxysmia: Diagnostic criteria. J Vestib Res. 2016;26(5-6):409-15. https://doi.org/10.3233/ves-160589

15. The National Multiple Sclerosis Society. Tegretol [Internet]. Disponível em: https://www.nationalmssociety.org/Treating-MS/ Medications/Tegretol

16. Almeida RG, Lyons DA. On Myelinated Axon Plasticity and Neuronal Circuit Formation and Function. J. Neurosci. 2017;37(42):10023-34. https://doi.org/10.1523/ JNEUROSCI.3185-16.2017

17. Gstoettner W, Swoboda H, Miiller C, Burian M. Preclinical detection of initial vestibulocochlear abnormalities in a patient with multiple sclerosis. Eur Arch Otorhinolaryngol. 1993;250(1):403. https://doi.org/10.1007/bf00176947 